\title{
28 Research Square \\ ESCO2 Inhibits p53 Transcription and Promotes \\ Proliferation and Migration of Lung Cancer Cells
}

Ming Liu

Tangdu Hospital Fourth Military Medical University: Air Force Medical University Tangdu Hospital

\section{Yinan Ma}

The First Affiliated Hospital of China Medical University

Enhua Wang ( $\nabla$ wangeh@hotmail.com )

College of Basic Medical Sciences and First Affiliated Hospital, China Medical University

Research

Keywords: ESCO2, NSCLC, p53, proliferation

Posted Date: May 11th, 2021

DOI: https://doi.org/10.21203/rs.3.rs-506320/v1

License: (1) This work is licensed under a Creative Commons Attribution 4.0 International License.

Read Full License 


\section{Abstract}

Purpose: This study aimed to reveal the expression of the Establishment of Sister Chromatid Cohesion Nacetyltransferase 2 (ESCO2) in non-small-cell lung carcinoma(NSCLC) and the relevant mechanisms.

Methods: ESCO2 expression and its correlation with the clinicopathological factors of NSCLC were evaluated via GEO database analysis and immunohistochemical staining. Transwell, MTT, and colonyforming assays were used to discover the effects of ESCO2 on cancer cell proliferation and migration. Moreover, Gene-Set Enrichment Analysis(GSEA) was employed to investigate the effects of ESCO2 on possible carcinogenic pathways.

Results: ESCO2 expression was found to be significantly higher in lung cancer tissues $(2+a n d 3+, 73.3 \%$, $96 / 131$; If added $1+, 90.1 \%, 118 / 131)$ than the adjacent tissues $(0.7 \%, 1 / 131)$. Besides, down-regulating ESCO2 expression could significantly inhibit the proliferation and migration of lung cancer cell lines (A549 and H460). Furthermore,p53 and its downstream target p21 were activated judging from the results of GSEA. However, transfection of siRNA-ESCO2 into the $\mathrm{H} 1299$ cell line without p53 resulted in no significant variations in terms of cell proliferation, migration, and colony formation, as well as the expression of p21 in these cells. Quantitative real-time PCR (qRT-PCR) assays proved that ESCO2 inhibits p53 expression during transcription. Co-immunoprecipitation(Co-IP)analyses indicated that ESCO2 can interact with histone methyltransferase SETDB1 (SET domain bifurcated histone lysine methyltransferase 1) in lung cells.

Conclusion: The amount of ESCO2 shows an abnormal increase in lung cancer cells compared to normal bronchial epithelial cells. Besides, ESCO2 can promote the malignancy of lung cancer cells by interfering with the $\mathrm{p} 53 / \mathrm{p} 21$ pathway.

\section{Introduction}

The cohesion of sister chromatids requires a series of conserved proteins (Smc1, Smc3, Mcd1/Scc1/Rad21) (1-4), as well as Eco1(Establishment of Cohesion 1)/Ctf7(Chromosome Transmission Fidelity7), an N-acetyltransferase member of the GNAT family and a crucial protein in the cellular S phase to build cohesion establishment (5-6). Eco1 can acetylate the cohesive subunit of Smc3, enabling the sister chromatids to be in a cohesive state (6-10). In vertebrates, two homologs of Eco1 are Esco1 and ESCO2. While these two homologs share similar reactivity, their contributions to the regulation of the cohesive functions vary, with ESCO2 as the more dominant (10). The absence of ESCO2 can lead to RBS syndrome, which appears as a loss of cohesion, genome instability, neural tube phenotype of microcephaly, craniofacial defects, and mental retardation (11).

ESCO2 is a differential gene that has been discovered in recent tumor bio-analysis studies. It has been found to correlate with poor prognosis in aggressive melanoma, breast cancer, renal cell carcinoma, and lung squamous cell carcinoma (12-16). Moreover, ESCO2 has been revealed to inhibit the metastasis of colon cancer by curbing the transcription of MMP2 (17) and promote the proliferation of gastric cancer 
cells by inhibiting p53 expression (18). However, few reports have focused on the relationship between ESCO2 and lung cancer, and the only one was about lung adenocarcinoma. In the article, the authors proposed that ESCO2, as an acetyltransferase, can acetylate lysine at position 277 of hnRNPA1 so that hnRNPA1 can continue to function in the nucleus as a regulator of PKM splicing, a promotor of PKM2 production, and an inhibitor of PKM1 production, therefore leading to the proliferation of lung adenocarcinoma cells (19). Previous literature has demonstrated that ESCO2 is related to the occurrence and development of tumors with various mechanisms of action. In this article, the relationships among ESCO2 expression, clinicopathological factors, and p53 transcription in NSCLC are described in detail.

\section{Materials And Methods Bioinformatics analysis}

LUAD gene expression data-set (GSE148036) was downloaded from the Gene Expression Omnibus database (https://www. ncbi. nlm. nih. gov/geo/query/acc. cgi?acc= GSE148036) for gene difference and GSEA analyses. Moreover, the Ualcan database (http://ual can. path. uab. edu) was employed to analyze the relationships among gene expression, clinical stages, and survival rates of NSCLC patients.

\section{Immunohistochemical staining}

Immunohistochemical staining was performed on several tissue microarrays (TMAs) with a thickness of $4 \mu \mathrm{m}$ (HLugA150CS03, HLugA030PG02, HLugS030PG02, OD-CT-RsLug03-002, Outdo Biotech, Shanghai, China). The slices were repaired in citrate buffer at a high temperature and pressure for 3 minutes. Subsequently, the primary antibody of ESCO2 (1:200, ab86003; Abcam) was added into the slices, followed by incubation at $4^{\circ} \mathrm{C}$ overnight. Immunoperoxidase labeling was performed with an $A B C D$ Kit(MTB, Fuzhou, China). Finally, DAB chromogen was added into the samples and the nuclei were dyed with hematoxylin.

Based on the percentage and the intensity of cell staining, ESCO2 expression levels were allocated into four categories, namely negative (no staining,-), weakly positive (only partially colored and light yellow granules,+), moderately positive (yellow granules, $2+$ ), and strongly positive (dark yellow or yellowish brown granules, $3+$ ). Since no standard to judge the positive immunohistochemical expression of ESCO2 is currently available and most of the lung cancer specimens (96/131) were categorized as level $2+$ or more, the expression levels of $2+$ and $3+$ were defined as positive, while the remaining two were defined as negative.

\section{Plasmid transfection and interference}

EX-NEG-M14 plasmids were obtained from GeneCopoeia Co. Ltd.. si-ESCO2 and negative control si-NC were purchased from Anhui General Bio Co. Ltd., and Lipofectamine 3000 (Invitrogen) was used to transfect cells.

\section{MTT and clone formation experiments}


After $24 \mathrm{~h}$ of transfection, a total of 3000 cells were cultured in $10 \%$ serum 1640 medium, and $20 \mu \mathrm{L}$ of MTT solution ( $5 \mathrm{mg} / \mathrm{ml}$ Thiazolyl Blue) was added to each plate. The absorbance numbers at $490 \mathrm{~nm}$ at $24 \mathrm{~h}, 48 \mathrm{~h}, 72 \mathrm{~h}$, and $96 \mathrm{~h}$ were detected and recorded by an automatic microplate reader. Another 800 cells contained in $4 \mathrm{ml}$ of $10 \%$ serum 1640 medium were cultured in a $6-\mathrm{cm}$ dish for 10 days. All cells were fixed in ice methanol and dyed with hematoxylin after the experiments.

\section{Transwell assay}

After 48 hours of transfection, cells were inoculated in the upper chamber at a density of $5 \times 10^{5}$ cells $/ 200 \mu \mathrm{L}$, and $600 \mu \mathrm{L}$ of $20 \%$ serum medium was added to the lower chamber. The cells were harvested after $24 \mathrm{~h}$ of inoculation and fixed in ice-methanol for 15 minutes, followed by hematoxylin staining. Ten fields of view were randomly selected, and the number of cells migrating to the lower chamber in each of the selected fields was counted.

\section{Western blot assay}

The extracted proteins were separated by SDS-PAGE, transferred to a polyvinylidene difluoride (PVDF) membrane, and incubated with the primary antibodies(Table S1). On the next day, the samples were incubated with the horseradish peroxidase (HRP), which was coupled with anti-rabbit/mouse secondary antibody, at $37^{\circ} \mathrm{C}$ for $2 \mathrm{~h}$. Enhanced chemiluminescence (ECL) detection reagents were used to visualize the results.

\section{qRT-PCR assay}

TRIzol and chloroform were used to extract total RNA from the treated A549 cells, Reverse transcription was carried out by a PrimeScript RT kit (TAKARA). A 20 $\mu$ L SYBR Green PCR SuperMix system (Transgen, Beijing, China) was used for DNA amplification, and the amplification cycle was $50{ }^{\circ} \mathrm{C}, 2 \mathrm{~min} ; 95^{\circ} \mathrm{C}, 10$ min; $95^{\circ} \mathrm{C}, 40 \mathrm{~s}$ for 40 cycles, and finally end by $60^{\circ} \mathrm{C}, 60 \mathrm{~s}$. $\beta$-actin was used as the control group, and the data were analyzed by $2-\Delta \Delta C T$.

\section{Immunoprecipitation}

The immunoprecipitation experiments were performed with the Immunoprecipitation Kit (Proteintech, USA) following the manufacturer's protocol. The experiment results were detected by Western blot.

\section{Statistical analysis}

Data analysis was performed on GraphPad Prism 8. 0. The chi-square test was used to analyze the correlation between clinicopathological factors and protein expression levels. The t-test was used to detect the gray value in the Western blot assay. $p<0.05$ was considered statistically significant.

\section{Results}




\section{ESCO2 is highly expressed in NSCLC and associated with poor prognosis}

According to the GEO database, the expression of ESCO2 mRNA in lung adenocarcinoma is significantly higher than in the adjacent tissues $(p<0.05$, Fig. 1A-B). Similar results were obtained from the Ualcan online database, which states that the high expression of ESCO2 is associated with later clinical stages and poor prognosis of lung adenocarcinoma $(p<0.05$, Fig. 1C-D).

To assess ESCO2 expression in tissues, 131 pairs of tissue microarrays (87 of adenocarcinoma, 44 of squamous cell carcinomas) were used for immunohistochemistry assay. ESCO2 was localized in the cytoplasm and the rate of positive ESCO2 expression in lung carcinoma was revealed as $73.3 \%(2+$ and3+,73. 3\%, 96/131; If added 1+,90.1\%,118/131). However, similar to the bioinformatic analysis, ESCO2 expression in adjacent lung tissues was quite low, with a positive rate of merely $0.7 \%(1 / 131$, Fig. 1E). Moreover, the positive rate of ESCO2 expression in lung adenocarcinoma (75. 9\%) was slightly higher than squamous cell carcinomas (68.2\%). Besides, the ESCO2 expression level in most adenocarcinoma samples was strongly positive $(+++, 56.3 \%, 49 / 87)$, while in most squamous cell carcinoma samples, the ESCO2 expression level was moderately positive $(++, 43.1 \%, 19 / 44)$. The positive expression of ESCO2 was correlated with late TNM stages and lymph node metastasis of NSCLC $(p=0.045, p=0.011$, respectively), but not with sex, age or differentiation ( $p>0.05$, Table 1$)$. 
Immunohistochemical staining analysis about the relationship between ESCO2 expression and clinicopathological factors in NSCLC.

\begin{tabular}{|c|c|c|c|c|c|}
\hline Clinicopathological & \multirow[t]{2}{*}{$\mathrm{n}$} & \multirow[t]{2}{*}{ Positive } & \multirow[t]{2}{*}{ Negative } & \multirow[t]{2}{*}{$x^{2}$} & \multirow[t]{2}{*}{$\mathrm{P}$} \\
\hline feature & & & & & \\
\hline \multicolumn{6}{|l|}{ Age (years) } \\
\hline$<60$ & 46 & 34 & 12 & \multirow[t]{2}{*}{0.158} & \multirow[t]{2}{*}{0.691} \\
\hline$\geq 60$ & 70 & 54 & 16 & & \\
\hline \multicolumn{6}{|l|}{ Gender } \\
\hline Male & 69 & 55 & 14 & \multirow[t]{2}{*}{1.542} & \multirow[t]{2}{*}{0.214} \\
\hline Female & 46 & 32 & 14 & & \\
\hline \multicolumn{6}{|l|}{ Histological type } \\
\hline Squamous cell carcinoma & 44 & 30 & 14 & \multirow[t]{2}{*}{0.880} & \multirow[t]{2}{*}{0.348} \\
\hline Adenocarcinoma & 87 & 66 & 21 & & \\
\hline \multicolumn{6}{|l|}{ Differentiation } \\
\hline Well & 40 & 31 & 9 & \multirow[t]{2}{*}{0.050} & \multirow[t]{2}{*}{0.822} \\
\hline Moderate\&Poor & 78 & 59 & 19 & & \\
\hline \multicolumn{6}{|l|}{ TNM classification } \\
\hline$\nabla+\nabla$ & 58 & 37 & 21 & \multirow[t]{2}{*}{3.995} & \multirow[t]{2}{*}{$0.045^{\star}$} \\
\hline$\nabla$ & 44 & 36 & 8 & & \\
\hline \multicolumn{6}{|l|}{ Lymph node metastasis } \\
\hline Positive & 55 & 33 & 22 & \multirow[t]{2}{*}{6.446} & \multirow[t]{2}{*}{$0.011^{\star \star}$} \\
\hline Negative & 47 & 39 & 8 & & \\
\hline
\end{tabular}

Notes: ${ }^{*} P<0.05,{ }^{*} \mathrm{P}<0.01 . \mathrm{n}=131$ lung cancer patients.

\section{Transfection of ESCO2 promotes the proliferation and migration of lung cancer cells}

The specific siRNA-ESCO2 sequence was transfected into the A549 and H460 cell lines to investigate the influence of ESCO2 on the proliferation and migration of lung cancer cells (Fig. 2A). MTT (Fig. 2B) and colony formation assays (Fig. 2D) showed that ESCO2 interference inhibited lung cancer cell proliferation of both cell lines (siNC $304 \pm 15$ VS siESCO2 128 \pm 10 , P凶0. 05 for A549 and siNC $232 \pm 15$ VS siESCO2 $66 \pm 8, P \bowtie 0.05$ for H460). Transwell assays (Fig. 2C) showed that ESCO2 interference could curb lung 
cancer migration (siNC $350 \pm 20$ VS siESCO2 225 \pm 15 , P凶0. 05 for A549 and siNC $225 \pm 14$ VS siESCO 2 $101 \pm 11$, Pख0. 05 for H460). However, ESCO2 transfection in the A549 cell line (Fig. 2E) promoted the

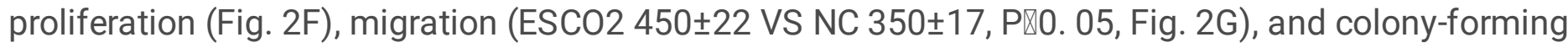
(ESCO2 $150 \pm 15$ VS NC $96 \pm 8$, Pø0. 05, Fig. $2 \mathrm{H}$ ) of the cell line.

\section{ESCO2 promotes the proliferation and migration of NSCLC by down-regulating p53}

To investigate the possible action mechanisms of ESCO2 in NSCLC, GSEA analysis was used to find out the possible pathways from the TCGA database. The plot showed ESCO2 may have a close relationship with the cell cycle and p53 pathway (Fig. 3A). Subsequently, the expressions of ESCO2 in A549 (wherein p53 is wild-type) and H460 cell lines were manually regulated, and Western blot was used to detect the expression of p53, as well as its downstream target of p21 and CDK1. The results showed that ESCO2 was negatively correlated with the expression of $\mathrm{p} 53$ and $\mathrm{p} 21$ while being positively correlated with CDK1 expression. (Fig. 3B). However, when transfecting siRNA-ESCO2 into the H1299 cell line (in which p53 was deficient), a similar phenomenon did not occur.

In order to clarify whether ESCO2 promotes the proliferation and migration of NSCLC by downregulating p53, siRNA-ESCO2 plasmids were transfected into the p53-deficient $\mathrm{H} 1299$ cell line (Fig. 4A). The results showed that the cell proliferation (Fig. 4B), migration (NC $270 \pm 20$ VS si-ESCO2 275 $\pm 15 ; p>0.05$, Fig. 4C), colony-forming (NC $150 \pm 8$ VS si-ESCO2 $136 \pm 13 ; p>0$. 05, Fig. 4D), and the concentration of p21 and CDK1 proteins did not reveal a significant change (Fig. 3B). The qRT-PCR results uncovered that ESCO2 overexpression could lead to a drop in the expression of p53 mRNA, ( $p<0.05$, Fig. 4E),suggesting that the inhibition of p53 by ESCO2 occurred during transcription. We suggested it exists a connection between ESCO2's transcriptional inhibitory function and histone methylation.Co-immunoprecipitation (CoIP) analyses indicated that ESCO2 can interact with histone methyltransferase SETDB1 in the A549 cell line (Fig. S1A). Twenty four hour after transfection,the A549 cells were treated with either DMSO or 100 $\mathrm{nm}$ chaetocin (a nonspecific inhibitor of histone lysine methyltransferases,Glpbio), the result showed treated with chaetocin could counteract ESCO2's inhibition of p53 and p21 (Fig. S1B).

\section{Discussion}

Firstly, this study proves that a high expression of ESCO2 protein is present in NSCLC tissues. In addition, the positive ESCO2 expression is correlated with late TNM stages and lymph node metastasis in NSCLC patients, and $\mathrm{ESCO} 2$ has been verified to promote the proliferation, colony formation, and migration of lung cancer cells. Specifically, after the transfection of siRNA-ESCO2 into A549 and H460 cell lines, the expression of p53/p21 increased while the cells revealed a significantly lower capability of proliferation and migration, but such phenomena were not found in the p53-deficient H1299 cell line, which was strongly consistent with conclusions of high p53 enrichment and the involvement of the cell circle pathways in the ESCO2 high-expression group in the GSEA plot. And finally, ESCO2 transfection could 
significantly inhibit the transcription from p53 genes, and ESCO2 was verified to promote malignancy in lung cancer cells through the p53/p21 pathway.

The action mechanisms of ESCO2 in tumors are not yet fully understood, and most previous reports focused on the highly conserved acetyltransferase domain at the C-terminal and the establishment of cohesion. On the other hand, the ESCO2 molecule also contains an $\mathrm{N}$-terminal composed of a disordered region and a $\mathrm{C} 2 \mathrm{H} 2$ zinc finger domain, making it a member of intrinsically unstructured proteins (IUPs). A disordered region cannot be folded into a clear and unique spatial structure. However, once these IUPs are combined with other functional proteins, a series of rapid interconversion conformations occur, creating an ordered structure that plays important biological functions in life activities (20). Previous studies have found that ESCO2 can bind to NICD with its disordered region, thereby inhibiting the enrichment of NICD on the Hes1 promoter to inhibit the Notch pathway (21). In this study, ESCO2 was revealed to curb the transcription of $\mathrm{p} 53$. Generally speaking, histone methylation in the promoter region leads to transcription inhibition. However, ESCO2 cannot methylate other molecules by itself, and only after mutations on the Cterminal (the acetyltransferase domain) are the ESCO2 molecules enabled for transcriptional repression (22). Such phenomenon could be attributed to the binding of ESCO2 to a certain methyltransferase (Fig. S1) because the functions of an IUP are dependent on its chaperone proteins (23). This preliminary study showed that ESCO2 interacts with the methyltransferase SETDB1, which is capable of methylating histones in the p53 promoter region (24). However, further studies are necessary to reveal the detailed mechanisms.

\section{Conclusion}

In summary,We have shown that ESCO2 serves as an oncology gene, which promotes the proliferation and migration of lung cancer cells. We proposed Esco2 inhibits p53 transcription and proved Esco2's role in promoting the malignant phenotype of lung cancer cells is achieved through p53 pathway.We suggested it exists a connection between ESCO2's transcriptional inhibitory function and histone methylation.

\section{Abbreviations}

Chaetocin: a nonspecific inhibitor of histone lysine methyltransferases

ESCO2: Establishment of Sister Chromatid Cohesion N-acetyltransferase2

GSEA: Gene Set Enrichment Analysis

NSCLC: Non-small cell lung cancer

RBS: Roberts Syndrome

SETDB1: SET domain bifurcated histone lysine methyltransferase 1 


\section{Declarations}

\section{Ethics approval and consent to participate}

Not applicable.

\section{Consent for publication}

Not applicable.

\section{Availability of data and materials}

All data generated or analyzed during this study are included in this article and its supplementary information files.

\section{Competing interests}

The authors declare that they have no competing interests.

\section{Funding}

This study was supported by the National Natural Science Foundation of China (Grant No. 81572854 and 81772489 to $\mathrm{EW})$

\section{Contributions}

ML conducted all experiments and made substantial contributions to the analysis. ML and YM drafted the manuscript. EW designed the study and revised the manuscript. All authors read and approved the final manuscript.

\section{Acknowledgements}

Not applicable

\section{References}

1. Itay Onn, Jill M Heidinger-Pauli, Vincent Guacci, et al. Sister Chromatid Cohesion: A Simple Concept with a Complex Reality. Ann Rev Cell Dev Biol. 2008;24: 105-129.

2. Skibbens RV. Establishment of Sister Chromatid Cohesion. Curr Biol. 2009;19: R1126-R1132.

3. Nasmyth K, Haering CH. Cohesion: Its Roles and Mechanisms. Ann Rev Genet. 2009;43: 525-558.

4. Uhlmann F. A matter of choice: The establishment of sister chromatid cohesion. EMBO Rep. 2009;10: 1095-1102.

5. Toth A, Ciosk R, Uhlmann F, et al. Yeast Cohesin complex requires a conserved protein, Eco1p(Ctf7), to establish cohesion between sister chromatids during DNA replication.Genes Dev. 1999;13: 320- 
333.

6. Ivanov D, Schleiffer A, Eisenhaber F, et al. Eco1 Is a Novel Acetyltransferase that Can Acetylate Protein Involved in Cohesion. Curr Biol. 2002;4: 323-328.

7. Lengronne A, Mclntyre J, Katou Y, et al. Establishment of Sister Chromatid Cohesion at the S. cerevisiae Replication Fork. Mol Cell. 2006;23: 787-799.

8. Unal E, Heidinger-Pauli JM, Kim W, et al. A Molecular Determinant for the Establishment of Sister Chromatid Cohesion. Science. 2008;321: 566-569.

9. Rolef Ben-Shahar T, Heeger S, Lehane C, et al. Eco1-Dependent Cohesin Acetylation During Establishment of Sister Chromatid Cohesion. Science. 2008;321: 563-566.

10. Alomer R. M., Eulália M L da Silva, Jingrong Chen, et al. Esco1 and Esco2 regulate distinct cohesin functions during cell cycle progression. Proc. Natl. Acad. Sci. U.S.A. 2017;12: 9906-9911.

11. Mengen E, Kotan LD, Ucakturk SA, et al. A Novel Frameshift Mutation in ESCO2 Gene in Roberts Syndrome. Coll Physicians Surg Pak. 2018;28:403-405.

12. Ryu B, Kim DS, Deluca AM,et al. Comprehensive expression profiling of tumor cell lines identifies molecular signatures of melanoma progression. PLoS One. 2007;2: e594.

13. Xiao B, Chen $L, K e Y$, et al. Identification of methylation sites and signature genes with prognostic value for luminal breast cancer[J]. BMC Cancer. 2018;18: 405.

14. Wang QL, Liu L. Establishment of cohesion 1 homolog 2 facilitates cell aggressive behaviors and induces poor prognosis in renal cell carcinoma.Clin Lab Anal. 2020;34: e23163.

15. Chen $\mathrm{H}$, Zhang L, He W,et al. ESCO2 knockdown inhibits cell proliferation and induces apoptosis in human gastric cancer cells. Biochem Biophys Res Commun. 2018;496: 475-81.

16. Weimin Zhang, Qichen Cui, Weifeng Qu, et al. TRIM58/cg26157385 methylation is associated with eight prognostic genes in lung squamous cell carcinoma.Oncol Rep. 2018;40: 206-216.

17. Xiong-Bo Guo, Bin Huang, Ying-Hua Pan, et al. ESCO2 inhibits tumor metastasis via transcriptionally repressing MMP2 in colorectal cancer. Cancer Manag Res.2018;10: 6157-6166.

18. Chen H, Zhang L, He W, et al. ESCO2 knockdown inhibits cell proliferation and induces apoptosis in human gastric cancer cells. Biochem Biophys Res Commun. 2018,496: 475-81.

19. Hui-er Zhu, Tao Li, Shengnan Shi, et al. ESCO2 promotes lung adenocarcinoma progression by regulating hnRNPA1 acetylation. Journal of Experimental \& Clinical Cancer Research. 2021;40:64.

20. Marín M, Uversky VN, Ott T. Intrinsic disorder in pathogen effectors: protein flexibility as an evolutionary hallmark in a molecular arms race. Plant Cell. 2013;25:3153-7

21. Leem YE, Choi HK, Jung SY,et al. Esco2 promotes neuronal differentiation by repressing Notch signaling. Cell Signal. 2011;23:1876-84.

22. Beom-Jun Kim, Kyung-Min Kang, Sung Yun Jung, et al. Esco2 is a novel corepressor that associates with various chromatin modifying enzymes. Biochemical and Biophysical Research Communications. 2008;372: 298-304. 
23. Janin J, Sternberg M J. Protein flexibility, not disorder, is intrinsic to molecular recognition. F1000 Biology Reports. 2013;5: 2-8.

24. Ogawa S, Fukud a A, Matsumoto Y,et al. SETDB1 Inhibits p53-Mediated Apoptosis and Is Required for Formation of Pancreatic Ductal Adenocarcinomas in Mice. Gastroenterology. 2020;159:682-696.

\section{Figures}

\section{Fig.1}

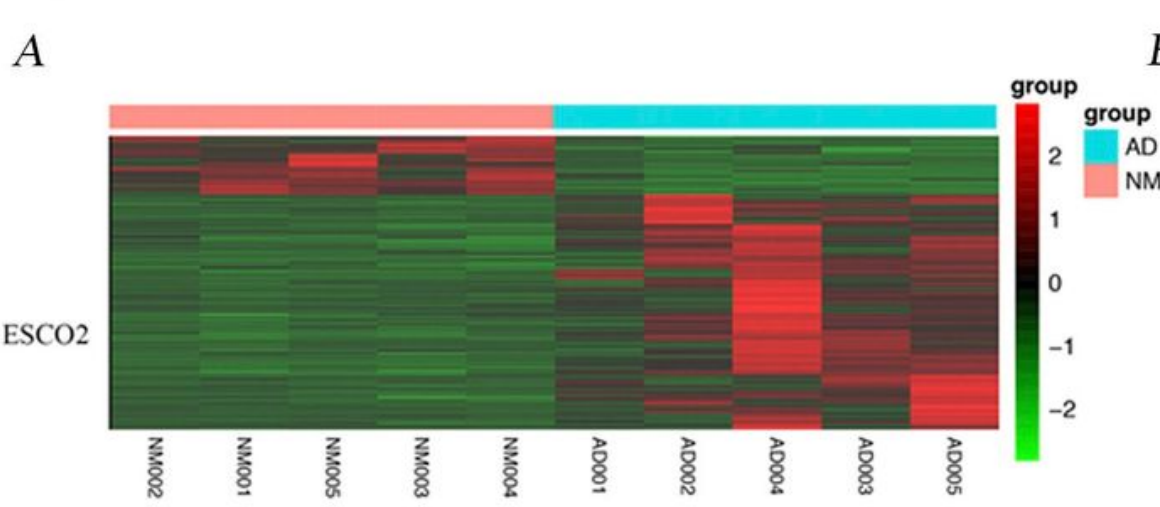

C



E

LUAD
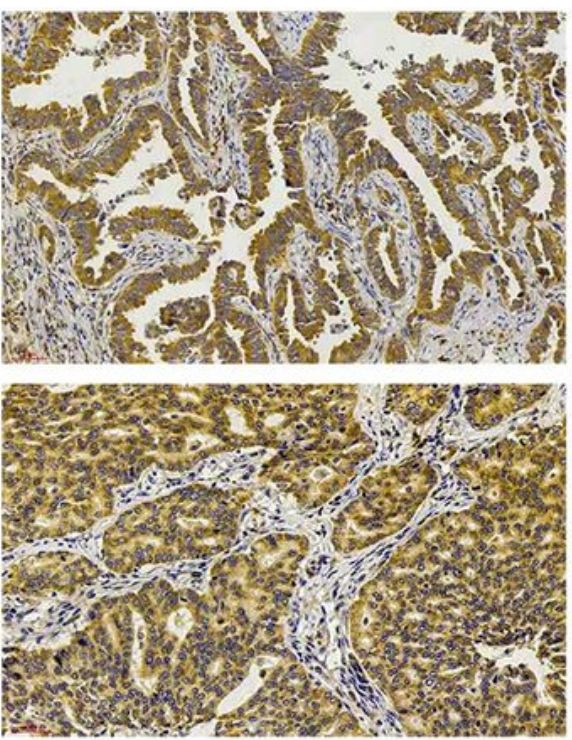

Expression of Esco2 in LUAD based on individual cancer stages

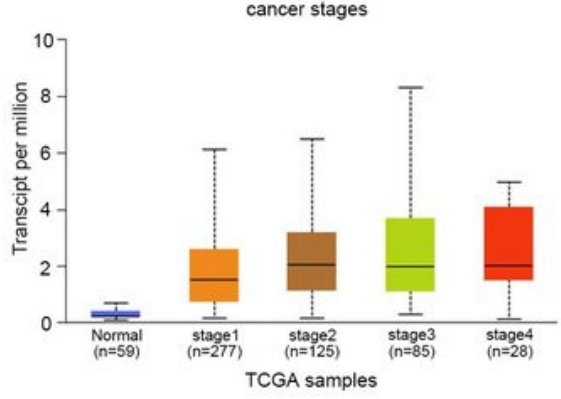

LUSC
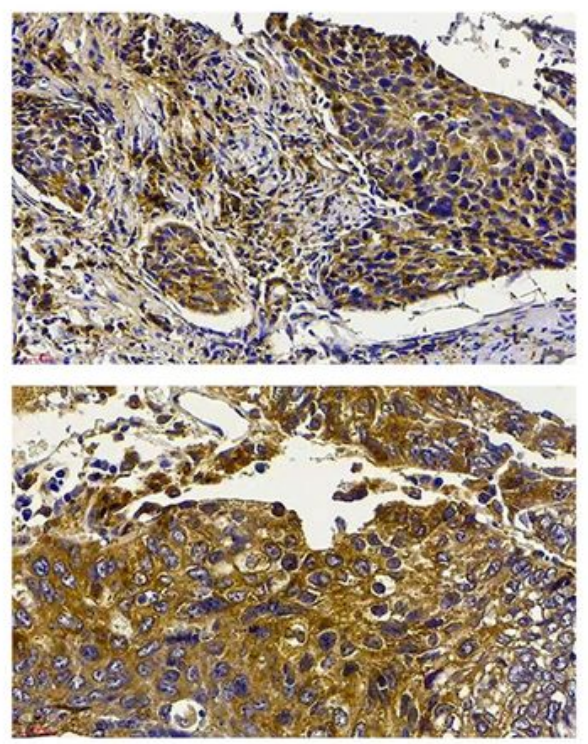

NM vs AD

D
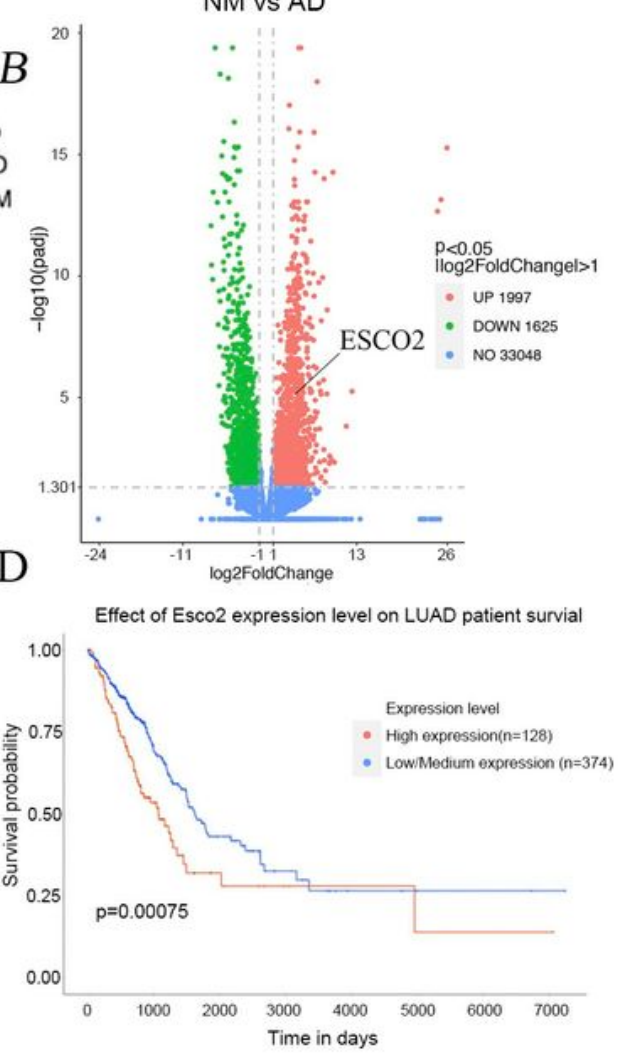

Normal Tissue

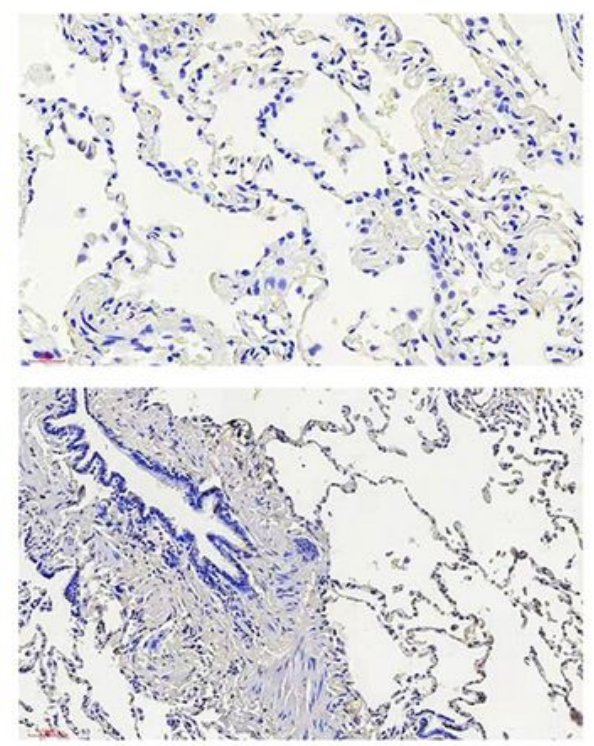




\section{Figure 1}

ESCO2 is highly expressed in NSCLC and associated with poor prognosis. A-B: ESCO2 is highly expressed in NSCLC and associated with poor prognosis. The expression of ESCO2 mRNA is significantly higher in LUAD tissues than normal ones in the GEO database. C-D: The Ualcan online database shows ESCO2 expression in LUAD tissues is positively correlated with late TNM stages (C) and poor prognosis (D). E: Immunohistochemical staining results show that ESCO2 is localized in the cytoplasm and the expressions of ESCO2 in lung adenocarcinoma and lung squamous cell carcinoma are significantly higher than the normal lung tissues. 
Fig.2
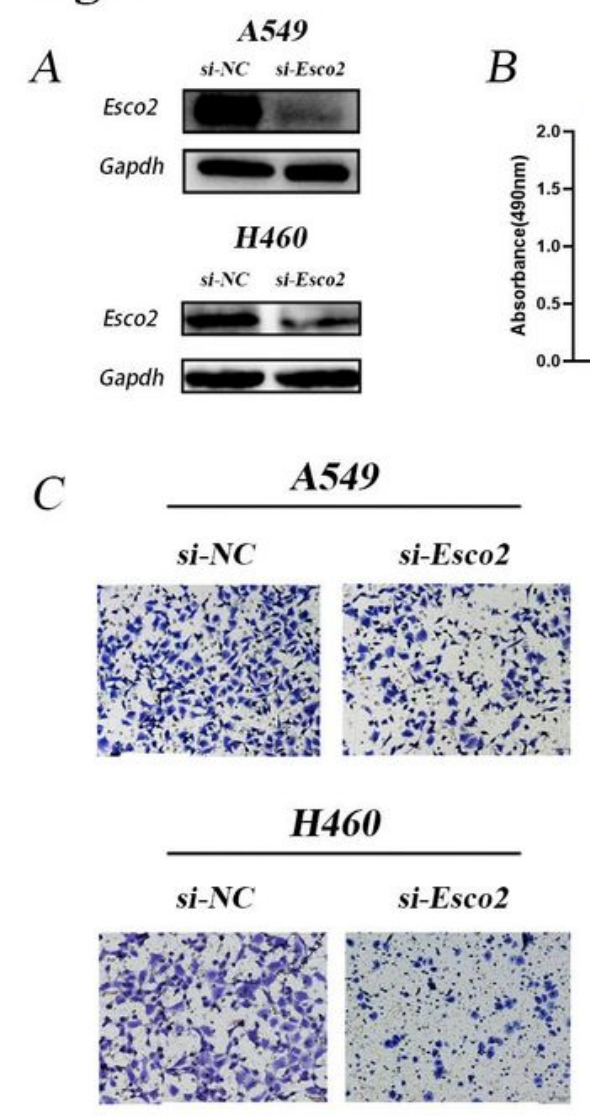

$E$

$$
F
$$
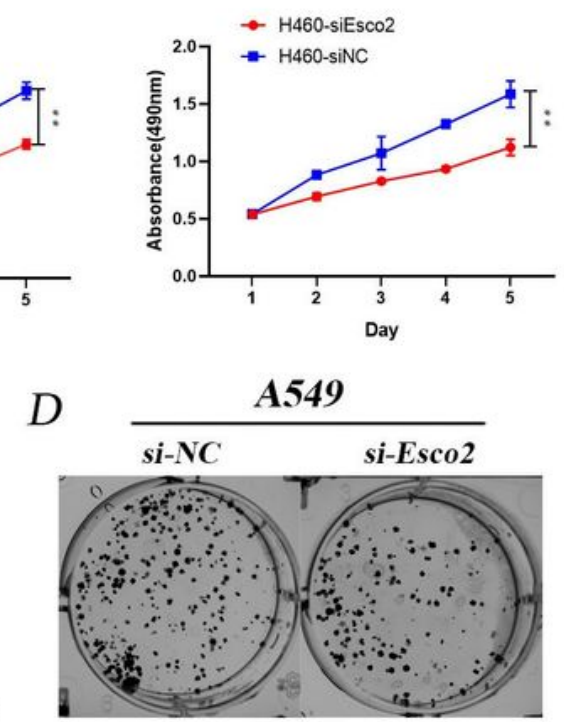

\section{H460}
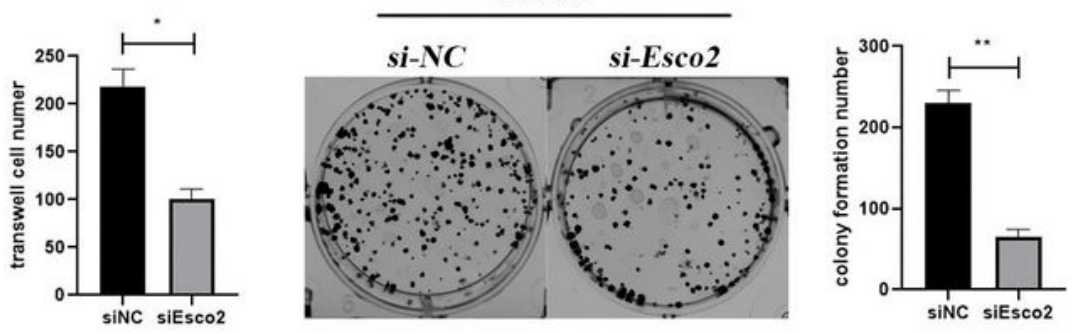


G
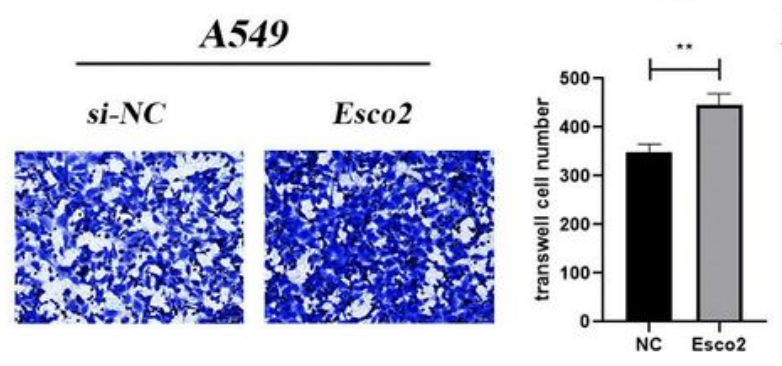

$H$
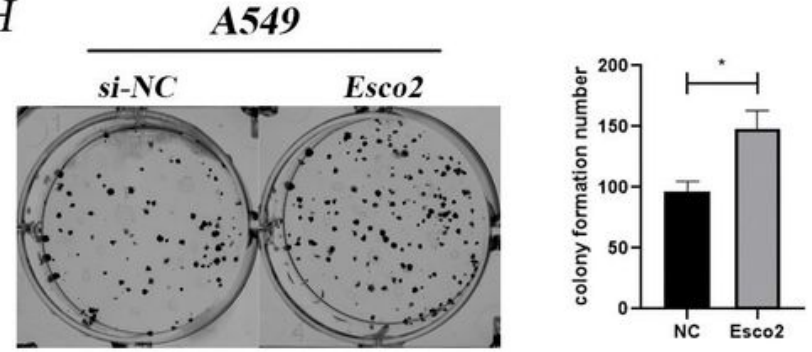

Figure 2

ESCO2 transfection promotes the proliferation and migration of lung cancer cells. A-D: ESCO2 interference in $\mathrm{A} 549$ and $\mathrm{H} 460$ cell lines (A) inhibits the proliferation (B), migration (C), and colonyforming (D). E-H: In contrast, the transfection of ESCO2 in the A549 cell line (E) promotes cellular proliferation $(F)$, migration $(G)$, and colony-forming $(H)$. 


\section{Fig.3}

A

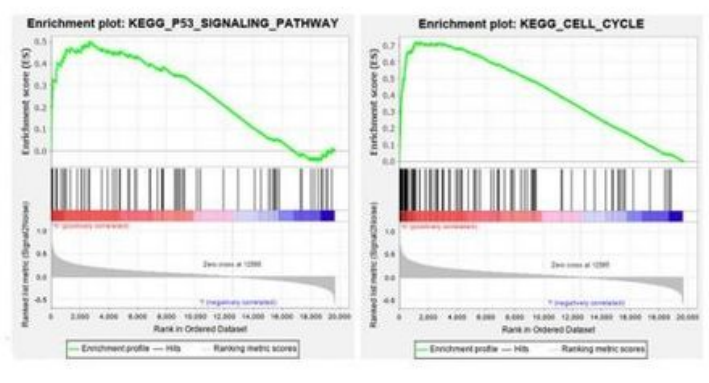

$B$

\begin{tabular}{ll}
$A 549 \quad H 460$ \\
\hline
\end{tabular}
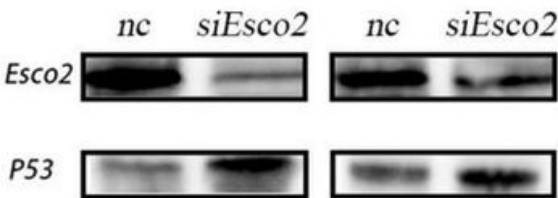

P21

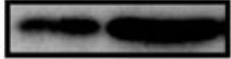

CDK

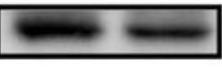

Gapdh
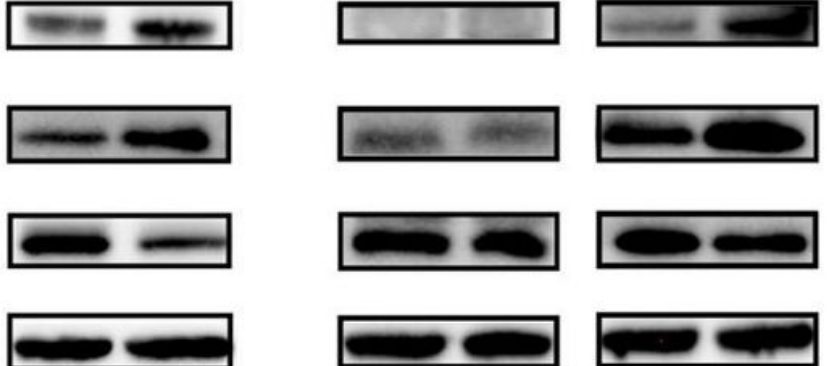

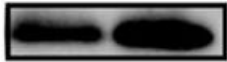

A549

$\operatorname{Esco} 2 \mathrm{nc}$
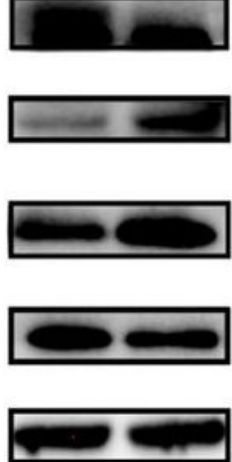

\section{Figure 3}

Esco2 inhibits the protein level of p53 A: Enrichment plots about p53 enrichments and cell circle pathways in the ESCO2 high-expression group in the TCGA LUAD cohort. B: Western blot analysis showed that ESCO2 suppression led to a decrease in CDK1 expression and an increase in p53 and p21 expressions in A549 (in which p53 is wild-type) and H460 cell lines. However, the above changes were not present in the p53-deficient $\mathrm{H} 1299$ cell line.

\section{Fig.4}

A

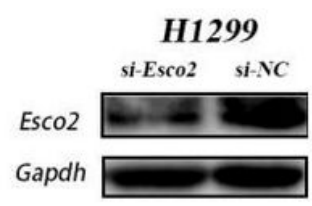

$B$

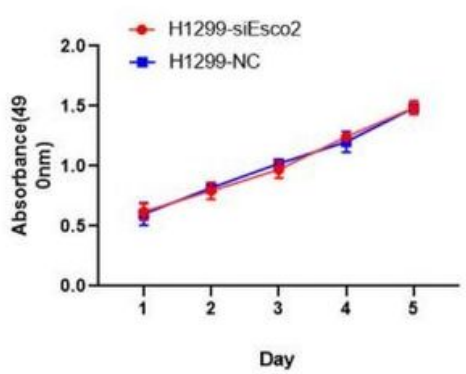

C
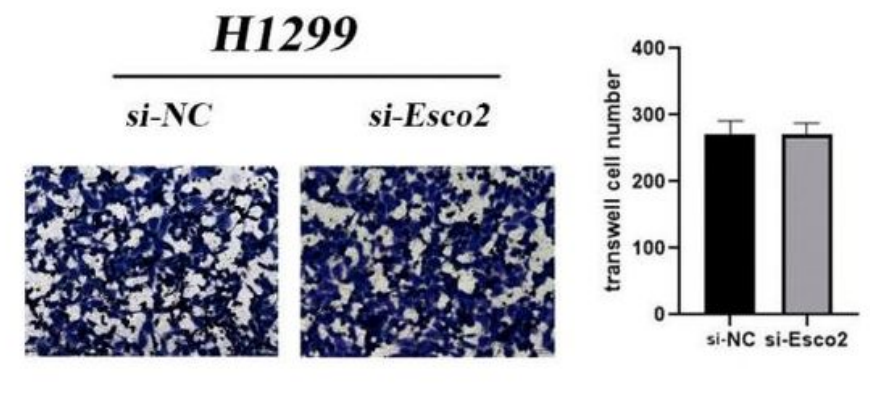

$D$

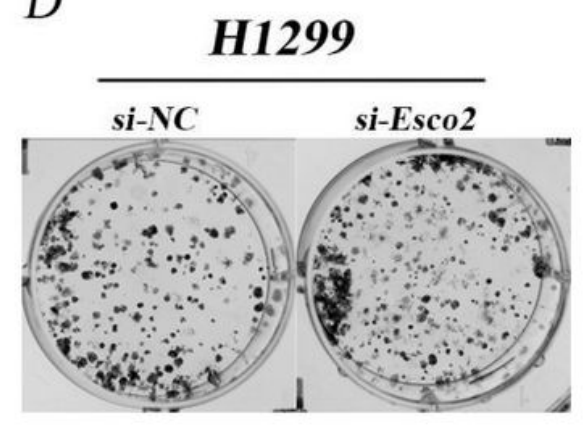

E
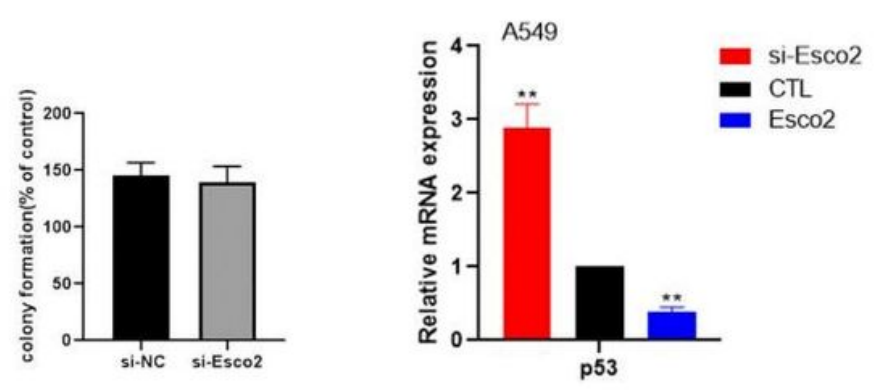
Figure 4

ESCO2 promotes the proliferation and migration of NSCLCs by downregulating p53. A-D: ESCO2

suppression (A) did not affect the proliferation (B), migration (C), and the colony-formation (D) of the p53deficient H1299 cell line. E: Furthermore, ESCO2 overexpression caused a decrease in the p53 mRNA expressions in the $\mathrm{A} 549$ cell line.

\section{Supplementary Files}

This is a list of supplementary files associated with this preprint. Click to download.

- Fig.S1.tif

- added.tif

- Tables1.docx 